\title{
A Trace Formula for Discrete Schrödinger Operators
}

By

\author{
Tomoyuki SHIRAI *
}

\begin{abstract}
We discuss two types of trace formula which arise from the inverse spectral problem for discrete Schrödinger operators as $L=-\Delta+V(x)$ where $V$ is a bounded potential. One is the relationshıp between a potential and spectral data, and another is the one between the green function of $L$ and periodic orbits of a state space.
\end{abstract}

\section{§1. Introduction}

The trace of the difference of two operators $L=-\Delta+V$ on $L^{2}\left(\mathbb{R}^{1}\right)$ and $L_{a}$ that is imposed the Dirichlet condition at $a \in \mathbb{R}^{1}$ has a relation

$$
\operatorname{Tr}\left(L-L_{a}\right)=V(a)=\lambda_{0}+\sum_{j=1}^{\infty}\left(\lambda_{2 j}+\lambda_{2 j-1}-2 \mu_{j}\right)
$$

for a periodic potential $V$, where $\left\{\lambda_{j}\right\}$ is the collection of all eigenvalues with periodic and anti-periodic boundary conditions. and $\left\{\mu_{j}\right\}$ is the collection of eigenvalues of certain Dirichlet Laplacian. It is the well known formula in Hill's theory for periodic Schrödinger operators. In [2], it has been extended to the class which is called reflectionless potential containing periodic potential. In [4], they studied systematically trace formulas by using the scattering quantity which is called the Krein's spectral shift function. We will show that similar results as these hold for a discrete Schrödinger operator $L$ on countable set and $L_{A}$ that is imposed the Dirichlet condition at a finite set $A$, that is,

Theorem 1.1. Let $G$ be a conntable sei and let $\Delta_{G}$ be a Laplacian on $G$. Let $V$ be a real-valued bounded function. Further, let $L=-\Delta_{G}+V$ and $L_{A}$ be imposed the Dirichlet condition on a finite set $A$. Then

$$
\frac{1}{|A|} \operatorname{Tr}\left(L-L_{A}\right)=\frac{1}{|A|} \sum_{a \in A} V(a)=\lambda_{\infty}-1-\int_{\lambda_{0}}^{\lambda_{\infty}} \theta_{A}(\lambda) d \lambda
$$

Communicated by T. Kawai, March 19. 1997. Revised November 14, 1997.

1991 Mathematics Subject Classification (s): 81U40, 58F20

* Supported by J.S.P.S. Research Fellowships for Young Scientists.

Research Institute for Mathematical Sciences, Kyoto University, Kyoto 606, Japan.

E-mail: shirai@kurims.kyoto-u.ac.jp 
where $\theta_{A}(\lambda)$ is a generalized Krein's spectral shift function.

Especially, if $G$ is $\mathbb{Z}^{1}$ and $A$ is a singleton $\{a\}$, then we can explicitly calculate of $\theta_{A}(\lambda)$, and the almost same relation as (1.1) holds.

In the area of quantum chaos, M. C. Gutzwiller has proposed the so-called Gutzwiller's trace formula [5]. It is the formula which connects the energy level (the spectrum of Schrödinger operators) with the classical periodic orbits. We will show that $\operatorname{Tr}\left(G_{\lambda}-G_{\lambda}^{A}\right)$ can be expanded by the periodic orbits on $A$ where $G_{\lambda}$ (resp. $G_{\lambda}^{A}$ ) is the resolvent of the operator $L$ (resp. $L_{A}$ ).

Theorem 1.2. There exists $\tilde{\lambda} \in \mathbb{R}$ such that for any $\lambda<\tilde{\lambda}$

$$
\begin{aligned}
\sum_{x \in G}\left(g_{\lambda}(x, x)-\right. & \left.g_{\lambda}^{A}(x, x)\right)=\sum_{a \in A} \frac{d}{d \lambda} \log g_{\lambda}(a, a) \\
& +\sum_{\gamma \in \Gamma} \frac{d S_{\gamma}(\lambda)}{d \lambda} \sum_{n \geq 1} \exp \left(-n S_{\gamma}(\lambda)-n \pi i L_{\gamma}\right)
\end{aligned}
$$

where $\Gamma$ is the set of all prime periodic orbits, $L_{\gamma}$ is the period of $\gamma$ and $S_{\gamma}(\lambda)$ is the length of a periodic orbit $\gamma$ with respect to the distance $d_{\lambda}$ defined by (4.1). formula.

It is thought as a discrete and heat version of the Gutzwiller's trace

\section{§ 2. A Trace Formula for the Inverse Spectral Problem}

Let $G$ be a countable set and $P=\{p(x, y)\}_{x, y \in G}$ a transition probability. We assume that the transition probability is (1) $m$-symmetric. (2) irreducible and (3) simple, i.e., (1) there exists a positive real-valued function $\{m(x)\}_{x \in G}$ on $G$ such that

$$
m(x) p(x, y)=m(y) p(y, x)
$$

for any $x, y \in G,(2)$ for any $x, y \in G$ there exists a positive integer $N$ such that

$$
p^{N}(x, y)>0
$$

and (3) $p(x, x)=0 .((3)$ is not essential but, for simplicity, we assume it.) Let $l^{2}(G, m)$ be an $l^{2}$-space with respect to the inner product given by

$$
\langle f, g\rangle=\sum_{x \in G} m(x) \overline{f(x)} g(x) .
$$

We define a discrete Laplacian on $l^{2}(G, m)$ as follows: for each $x \in G$.

$$
\Delta_{G} \phi(x)=\sum_{r \in G} p(x, r) \phi(r)-\phi(x) .
$$

Let $V$ be a real-valued bounded function and we define a discrete Schrödinger operator by 


$$
L=-\Delta_{G}+V \text {. }
$$

It is a linear bounded self-adjoint operator on $l^{2}(G, m)$.

Let $A \subset G$ be a finite subset of $G$. We consider two problems for our operator, i.e., one is

and the other is

$$
L \phi(x)=\lambda \phi(x) \quad x \in G
$$

$$
\left\{\begin{array}{l}
L_{A} \phi(x)=L \phi(x)=\lambda \phi(x) \quad x \in G \backslash A \\
L_{A} \phi(a)=0 \quad a \in A,
\end{array}\right.
$$

and their domains are $D(L)=l^{2}(G, m)$ and $D\left(L_{A}\right)=\left\{f \in l^{2}(G, m) ; f(a)=0\right.$ for any $a \in A$ \}. We denote the fundamental solutions of the associated heat equations by $p^{V}(t, x, y)$ and $p_{A}^{V}(t, x, y)$, respectively, and the associated green functions, that is, the integral kernels of $(L-\lambda)^{-1}$ and $\left(L_{A}-\lambda\right)^{-1}$ by $g_{\lambda}(x, y)$ and $g_{\lambda}^{A}(x, y)$, respectively. Remark that in general our heat kernels and green functions are not symmetric functions.

From now on, we assume that there exists a positive integer $M$ such that

$$
\sup _{x \in G}|\{r \in G ; p(x, r)>0\}| \leq M
$$

where $|K|$ is the cardinality of a set $K$. We can regard $G$ as an infinite graph. then the assumption (2.1) means that the maximum degree is bounded.

To show our trace formula we calculate the trace $\sum_{x \in G}\left(p^{V}(t, x, x)-p_{A}^{V}(t, x\right.$. $x)$ ) in two different ways. We use the following lemma for the first half of the trace formula.

Lemma 2.1. Let $\left\{w_{t}, P_{x}\right\}$ be a continuous time random walk with the generator $\Delta_{G}$, and $T_{A}$ the first hitting time to the set $A$. Then, as $\mapsto 0$,

$$
E_{a}\left[\delta_{a}\left(u^{\prime}\right)\right]=1-t+O\left(t^{2}\right)
$$

and

$$
\sum_{x \in G \backslash A} E_{x}\left[\delta_{x}\left(w_{t}\right) ; T_{A} \leq t\right]=O\left(t^{2}\right)
$$

where $\delta_{x}(\cdot)$ is the indicator function of $x \in G$,

Proof. Firstly, since $\Delta_{G}$ is the generator of $w_{t}$ and is bounded, we have

$$
\begin{aligned}
E_{a}\left[\delta_{a}\left(w_{t}\right)\right] & =\sum_{n \geq 0} \frac{t^{n}}{n !} \Delta_{G}{ }^{n} \delta_{a}(a) \\
& =1+t\left(\Delta_{G} \delta_{a}\right)(a)+O\left(t^{2}\right) \\
& =1-t+O\left(t^{2}\right)
\end{aligned}
$$

as $\mapsto 0$.

Secondly, we define a metric on $G$ as follows: for any $x, y \in G$ 


$$
d(x, y)=\inf \left\{\begin{aligned}
n \geq 0 ; & \exists \text { path } x=x_{0} x_{1 \ldots} x_{n}=y \\
& \text { s.t. } p\left(x_{i}, x_{i+1}\right)>0 \quad 0 \leq \forall i \leq n-1
\end{aligned}\right\} .
$$

Put $M \geq 1$ as the assumption (2.1). Then it is obvious that the cardinality of a set $\{x \in G ; d(x, A)=n\}$ is less than $|A| M^{n}$. Then we obtain

$$
\begin{aligned}
& \sum_{x \in G \backslash A} E_{x}\left[\delta_{x}\left(w_{t}\right) ; T_{A} \leq t\right]=\sum_{n \geq 1} \sum_{\substack{x \in G \\
d(x, A)=n}} E_{x}\left[\delta_{x}\left(w_{t}\right) ; T_{A} \leq t\right] \\
\leq & \sum_{n \geq 1}|A| M^{n} P_{x}[w \text { has at least } 2 n \text { jumps up to time } t] \\
= & \sum_{n \geq 1}|A| M^{n} \sum_{k \geq 2 n} \frac{e^{-t} t^{k}}{k !} \leq \sum_{n \geq 1}|A| M^{n} t^{2 n} \leq C t^{2} \quad \text { as } t \rightarrow 0 .
\end{aligned}
$$

Here we used the fact that the number of jumps of the random walk up to time $t$ obeys the Poisson law with mean 1 .

Now we show the first half of the trace formula.

Proposition 2.2. Let $V(x)$ be a real-valued bounded function on $G$. Then,

$$
\sum_{x \in G}\left(p^{V}(t, x, x)-p_{A}^{V}(t, x, x)\right)=|A|-t\left(\sum_{a \in A} V(a)+|A|\right)+O\left(t^{2}\right) \text { as } t \rightarrow 0
$$

where $|A|$ is the cardinality of the set $A$.

Proof. By the Feynman-Kac formula, we have

$$
p^{V}(t, x, x)-p_{A}^{V}(t, x, x)=E_{x}\left[e^{-\int_{0}^{t} V\left(w_{s}\right) d s}\left(1-\chi_{\left\{T_{A}>t\right\}}\right) \delta_{x}\left(w_{t}\right)\right]
$$

where $\chi_{\left\{T_{A}>t\right\}}$ is the indicator function of a set $\left\{T_{A}>t\right\}$. We consider the trace of the difference of two heat kernels

$$
\begin{aligned}
\sum_{x \in G}( & \left.p^{V}(t, x, x)-p_{A}^{V}(t, x, x)\right) \\
\quad= & \sum_{x \in G} E_{x}\left[e^{-\int_{0}^{t} V\left(w_{s}\right) d s}\left(1-\chi_{\left\{T_{A}>t\right\}}\right) \delta_{x}\left(w_{t}\right)\right] \\
\quad= & \sum_{x \in G} \sum_{n \geq 0} \frac{(-1)^{n}}{n !} E_{x}\left[\left(\int_{0}^{t} V\left(w_{s}\right) d s\right)^{n}\left(1-\chi_{\left\{T_{A}>t_{s}\right.}\right) \delta_{x}\left(w_{t}\right)\right] .
\end{aligned}
$$

For $n=0$, by using Lemma 2.1 we have

$$
\begin{aligned}
& \sum_{x \in G} E_{x}\left[\left(1-\chi_{\left\{T_{A}>t\right\}}\right) \delta_{x}\left(w_{t}\right)\right] \\
& =\sum_{a \in A} E_{a}\left[\delta_{a}\left(w_{t}\right)\right]+\sum_{x \in G \backslash A} E_{x}\left[\delta_{x}\left(w_{t}\right) ; T_{A} \leq t\right] \\
& =|A|(1-t)+O\left(t^{2}\right) \quad \text { as } t \rightarrow 0 .
\end{aligned}
$$

For $n=1$, we have 


$$
\begin{gathered}
\sum_{x \in G} E_{x}\left[\left(\int_{0}^{t} V\left(w_{s}\right) d s\right)\left(1-\chi_{\left\{T_{A}>t\right\}}\right) \delta_{x}\left(w_{t}\right)\right] \\
=\int_{0}^{t} d s \sum_{x \in G} \sum_{y \in G}\left(p^{0}(s, x, y) V(y) p^{0}(t-s, y, x)\right. \\
\left.-p_{A}^{0}(s, x, y) V(y) p_{A}^{0}(t-s, y, x)\right)
\end{gathered}
$$

where $p^{0}(t, x, y)$ and $p_{A}^{0}(t, x, y)$ are the heat kernels for the case that the potential $V$ is identically zero. Using the semigroup property, we have

$$
\begin{aligned}
& =\int_{0}^{t} d s \sum_{y \in G} V(y)\left(p^{0}(t, y, y)-p_{A}^{0}(t, y, y)\right) \\
& =t\left\{\sum_{a \in A} V(a) E_{a}\left[\delta_{a}\left(w_{t}\right)\right]+\sum_{y \in G \backslash A} V(x) E_{x}\left[\delta_{x}\left(w_{t}\right) ; T_{A} \leq t\right]\right\} \\
& =t \sum_{a \in A} V(a)+O\left(t^{2}\right) \quad \text { as } t \rightarrow 0 .
\end{aligned}
$$

Last we estimate the term for $n \geq 2$.

$$
\begin{aligned}
& \left|\sum_{n \geq 2} \frac{(-1)^{n}}{n !} \sum_{x \in G} E_{x}\left[\left(\int_{0}^{t} V\left(w_{s}\right) d s\right)^{n}\left(1-\chi_{\left\{T_{A}>t\right\}}\right) \delta_{x}\left(w_{t}\right)\right]\right| \\
& \quad \leq \sum_{n \geq 2} \frac{t^{n}}{n !}\|V\|_{\infty}^{n} \sum_{x \in G} E_{x}\left[\left(1-\chi_{\left\{T_{A}>t\right\}}\right) \delta_{x}\left(w_{t}\right)\right] \\
& \quad \leq C t^{2} .
\end{aligned}
$$

Then, we have

$$
\sum_{x \in G}\left(p^{V}(t, x, x)-p_{A}^{V}(t, x, x)\right)=|A|-t\left(\sum_{a \in A} V(a)+|A|\right)+O\left(t^{2}\right) \quad \text { as } t \rightarrow 0 .
$$

Next we will calculate the difference of two green functions for the second half of the trace formula. Before doing that, we prepare a lemma.

Lemma 2.3. Let $G_{\lambda}^{A}$ be a $|A| \times|A|$ matrix with the elements $\left(G_{\lambda}^{A}\right)_{a, b}=$ $g_{\lambda}(a, b)$ for $a, b \in A$. Then $\operatorname{det} G_{\lambda}^{A}$ is holomorphic in $\lambda \in \mathbb{C} \backslash \sigma(L)$. Moreover, for $\lambda$ $\in \mathbb{C} \backslash\left[\lambda_{0}, \lambda_{\infty}\right]$, the determinant $\operatorname{det} G_{\lambda}^{A}$ is non-zero, where $\sigma(L)$ is the spectral set of the operator $L, \lambda_{0}=\inf \sigma(L)$ and $\lambda_{\infty}=\sup \sigma(L)$.

Proof. Note that $g_{\lambda}(x, y)$ is holomorphic in $\lambda \in \mathbf{C} \backslash \sigma(L)$. It is obvious by the definition of the determinant that $\operatorname{det} G_{\lambda}^{\mathrm{A}}$ is also holomorphic in $\lambda \in \mathbf{C} \backslash \sigma(L)$. Recall that 


$$
g_{\lambda}(x, y)=\left(\frac{m(y)}{m(x)}\right)^{1 / 2} \int_{\sigma(L)} \frac{1}{\xi-\lambda} d\left\langle E(\xi) e_{x, e_{y}}\right\rangle
$$

where $\left\{e_{x}\right\}_{x \in G}$ is an orthonormal basis of $l^{2}(G, m)$ such that

$$
e_{x}(y)=\left\{\begin{array}{cl}
m(x)^{-1 / 2} & \text { if } y=x \\
0 & \text { otherwise }
\end{array}\right.
$$

and $E(\xi)$ is the resolution of the identity for the operator $L$. Let $f_{0}$ be an $|A|$ dimensional vector such that $\left\|f_{0}\right\|_{A}=1$, where $\langle\circ,\rangle_{A}$ is the inner product of $l^{2}(A, m)$. Let $f \in l^{2}(G, m)$ be the extension of $f_{0}$ such that $\operatorname{supp} f \subset A, f(a)=$ $f_{0}(a)$ for any $a \in A$ and $\|f\|=1$. Then we have

$$
\left\langle f_{0}, G_{\lambda}^{A} f_{0}\right\rangle_{A}=\left\langle f, G_{\lambda} f\right\rangle=\int_{\sigma(L)} \frac{1}{\xi-\lambda} d \mu_{f}(\xi)
$$

where $d \mu_{f}(\xi)=d\|E(\xi) f\|^{2}$. We will estimate $\left|\left\langle f, G_{\lambda} f\right\rangle\right|$ from below. Firstly, in the case that $|\operatorname{Im} \lambda|>0$, for any $f \in l^{2}(G, m)$, we have

$$
\left|\left\langle f . G_{\lambda} f\right\rangle\right| \geq\left|\int_{\sigma(L)} \frac{\operatorname{Im} \lambda}{|\xi-\lambda|^{2}} d \mu_{f}(\xi)\right|
$$

$$
\geq \frac{|\operatorname{Im} \lambda|}{\max _{\xi \in \sigma(L)}|\xi-\lambda|^{2}}
$$

Secondly, when $\lambda \in \mathbb{R} \backslash\left[\lambda_{0}, \lambda_{\infty}\right]$, we have

$$
\left|\left\langle f, G_{\lambda} f\right\rangle\right| \geq \frac{1}{\max } \frac{1}{\left(\left|\lambda-\lambda_{0}\right|,\left|\lambda-\lambda_{\infty}\right|\right)} .
$$

In both cases, there exists a positive constant $C(\lambda)$ depending only on $\lambda$ such that $\left|\left\langle G_{\lambda} f, f\right\rangle\right| \geq C(\lambda)>0$. Then, for any $\lambda \in \mathbb{C} \backslash\left[\lambda_{0}, \lambda_{\infty}\right]$, $\operatorname{det} G_{\lambda}^{A} \neq 0$.

Remark 2.4. For $\lambda \in\left[\lambda_{0}, \lambda_{\infty}\right] \cap \sigma(L)^{c}$, the $\operatorname{determinant} \operatorname{det} G_{\lambda}^{A}$ may vanish.

Lemma 2.5. $\quad \lambda \in \mathbb{C} \backslash\left[\lambda_{0}, \lambda_{\infty}\right]$. Then for any $x, y \in G$

$$
g_{\lambda}(x, y)-g_{\lambda}^{A}(x, y)=\sum_{a \in A} g_{\lambda}(x, a)\left(G_{\lambda}^{A}\right)^{-1} g_{\lambda}(a, y)
$$

where $\left(G_{\lambda}^{A}\right)^{-1}$ acts on the first variable.

Proof. Let $F_{\lambda}(t)=F_{\lambda}(t, w)=\int_{0}^{t}\left(-\lambda+V\left(w_{t}\right)\right) d t$. If $\lambda<\inf _{x \in G} V(x), F_{\lambda}(t)$ $>0$, and so $F_{\lambda}(\infty)=\infty$. For any $\lambda<\inf _{x \in G} V(x)$. by the strong Markov property, we have

$$
g_{\lambda}(x, y)-g_{\lambda}^{A}(x, y)=E_{x}\left[\int_{T_{4}}^{\infty} e^{-F_{\lambda}(t)} \delta_{y}\left(w_{t}\right) d t\right]
$$




$$
\begin{aligned}
& =E_{x}\left[e^{-F_{\lambda}\left(T_{A}\right)} E_{x}\left[\int_{0}^{\infty} e^{-F_{\lambda}\left(t, S_{T_{A}} w_{t}\right)} \delta_{y}\left(S_{T_{A}} w_{t}\right) d t \mid \mathscr{F}_{T_{A}}\right] ; T_{A}<\infty\right] \\
& =E_{x}\left[e^{-F_{\lambda}\left(T_{A}\right)} E_{w_{T_{A}}}\left[\int_{0}^{\infty} e^{-F_{A}(t)} \delta_{y}\left(w_{t}\right) d t\right]: T_{A}<\infty\right]=E_{x}\left[e^{-F_{A}\left(T_{A}\right)} g_{\lambda}\left(w_{T_{A}}, y\right) ; T_{A}<\infty\right]
\end{aligned}
$$

where $\left(S_{s} u^{\prime}\right)_{t}=w_{t+s}$. We put $\mu_{x, \lambda}(a)=E_{x}\left[e^{-F_{\lambda}\left(T_{A}\right)}: w_{T_{A}}=a . T_{A}<\infty\right]$ for each $a \in$ A. Then,

$$
g_{\lambda}(x, y)-g_{\lambda}^{A}(x, y)=\sum_{a \in A} g_{\lambda}(a, y) \mu_{x, \lambda}(a)
$$

Next, in the same way as above, we have

$$
g_{\lambda}(x, a)=\sum_{b \in A} g_{\lambda}(b, a) \mu_{x, \lambda}(b)
$$

for each $x \in G$ and $a \in A$.

By Lemma 2.3, there exists an inverse matrix of $G_{\lambda}^{A}$. Then we have

$$
\begin{aligned}
& \sum_{a \in A} g_{\lambda}(x, a)\left(G_{\lambda}^{A}\right)^{-1} g_{\lambda}(a, y) \\
& =\sum_{a \in A} \sum_{b \in A} g_{\lambda}(b, a) \mu_{x, \lambda}(b)\left(G_{\lambda}^{A}\right)^{-1} g_{\lambda}(a, y) \\
& =\sum_{b \in A} \mu_{x, \lambda}(b) \sum_{a \in A} g_{\lambda}(b, a)\left(G_{\lambda}^{A}\right)^{-1} g_{\lambda}(a, y) \\
& =\sum_{b \in A} \mu_{x, \lambda}(b) g_{\lambda}(b, y)=g_{\lambda}(x, y)-g_{\lambda}^{A}(x, y) .
\end{aligned}
$$

The lemma is obtained by analytic continuation.

Proposition 2.6. Let $\lambda \in \mathbb{C} \backslash\left[\lambda_{0}, \lambda_{\infty}\right]$. Then

$$
\sum_{x \in G}\left(g_{\lambda}(x, x)-g_{\lambda}^{-\lambda}(x, x)\right)=\frac{d}{d \lambda} \log \operatorname{det} G_{\lambda}^{A} .
$$

Proof. Since $\left(G_{\lambda}^{A}\right)^{-1}$ is a linear operator, taking summation over $x \in G$, we have

$$
\begin{aligned}
\sum_{x \in G}\left(g_{\lambda}(x, x)-g_{\lambda}^{A}(x, x)\right) & =\sum_{x \in G} \sum_{a \in A} g_{\lambda}(x, a)\left(G_{\lambda}^{A}\right)^{-1} g_{\lambda}(a, x) \\
& =\sum_{a \in A}\left(G_{\lambda}^{A}\right)^{-1} \frac{d}{d \lambda} G_{\lambda}^{A}(a, a)=\operatorname{Tr}\left(\left(G_{\lambda}^{A}\right)^{-1} \frac{d}{d \lambda} G_{\lambda}^{A}\right) \\
& =\frac{d}{d \lambda} \log \operatorname{det} G_{\lambda}^{A} .
\end{aligned}
$$


Here we used the fact that $\frac{d}{d \lambda}(L-\lambda)^{-1}=(L-\lambda)^{-2}$ and $\operatorname{det} G_{\lambda}^{A}$ is non-zero in $\lambda$ $\in \mathbb{C} \backslash\left[\lambda_{0}, \lambda_{\infty}\right]$ by Lemma 2.3 .

Next we define a generalized Krein's spectral shift function $\theta_{A}(\lambda)$. Recall that for any $f \in l^{2}(G, m),\left\|\left(\lambda G_{\lambda}+I\right) f\right\| \rightarrow 0$ as $|\lambda| \rightarrow \infty$. Then, since $G_{\lambda}^{A}$ is a finite dimensional matrix, we have

$$
\left\|\lambda G_{\lambda}^{A}+I\right\| \rightarrow 0 \text { as }|\lambda| \rightarrow \infty .
$$

Therefore because of the continuity of the determinant, for $\operatorname{Im} \lambda>0$

$$
\operatorname{det} G_{\lambda}^{A} \sim(-\lambda)^{-|A|} \text { as }|\lambda| \rightarrow \infty .
$$

We take the branch of the logarithm so that $\operatorname{Im} \log \operatorname{det} G_{\lambda+t \epsilon}^{A} \rightarrow 0$ as $\lambda \rightarrow-\infty$. Let $\left\{\nu_{k}(\lambda)\right\}_{k=1}^{|A|}$ be eigenvalues of $G_{\lambda}^{A}$. Then, $\operatorname{Im} \log \operatorname{det} G_{\lambda}^{A}=\sum_{k=1}^{A} \operatorname{Im} \log \nu_{k}(\lambda)$. On the other hand, for each eigenvalue $\nu_{k}(\lambda)$, there exists a normalized eigenfunction $f_{k}$ such that

$$
\nu_{k}(\lambda)=\left\langle f_{k}, G_{\lambda}^{\mathrm{A}} f_{k}\right\rangle_{A}=\int_{\sigma(L)} \frac{1}{\xi-\lambda} d \mu_{f_{k}}(\xi) .
$$

Here we used (2.4). Then for any $\operatorname{Im} \lambda>0$ and $1 \leq k \leq|A|, \operatorname{Im} \nu_{k}(\lambda)>0$, and since the unordered tuple of eigenvalues is continuous in $\lambda$, by the way of taking the branch of the logarithm, we have

$$
0<\operatorname{Im} \log \operatorname{det} G_{\lambda}^{A}<|A| \pi
$$

Hence, by the Fatou's theorem, a limit

$$
\theta_{A}(\lambda):=\lim _{\epsilon \rightarrow 0} \frac{1}{\pi|A|} \operatorname{Im} \log \operatorname{det} G_{\lambda+t \epsilon}^{A}
$$

exists for almost every $\lambda \in \mathbb{R}$ and $0 \leq \theta_{A}(\lambda) \leq 1$. We call it a generalized Krein's spectral shift function.

Lemma 2.7. For almost every $\lambda \in \mathbb{R}, \theta_{A}(\lambda)$ exists and $0 \leq \theta_{A}(\lambda) \leq 1$. In particular.

$$
\theta_{A}(\lambda)= \begin{cases}0 & \text { if } \lambda<\lambda_{0} \\ 1 & \text { if } \lambda>\lambda_{\infty} .\end{cases}
$$

Proof. We have already shown the existence and so we will show only the second statement. Since $\operatorname{det} G_{\lambda}^{A}$ is real-valued for $\lambda \in \mathbb{R} \backslash\left[\lambda_{0}, \lambda_{\infty}\right]$, by the definition of the $\theta_{A}(\lambda)$, we have

$$
\theta_{A}(\lambda) \in\left\{\frac{k}{|A|} ; k \in \mathbb{Z}\right\}
$$


For any $x, y \in G$, the convergence of the green function $g_{\lambda+i \epsilon}(x, y)$ as $\epsilon \rightarrow 0$ is uniform on an arbitrary compact set $K \subset \mathbf{R} \backslash\left[\lambda_{0}, \lambda_{\infty}\right]$. Then, as $\epsilon \rightarrow 0$, Im log det $G_{\lambda+i \epsilon}^{A}$ also converges uniformly on compact sets in $\mathbb{R} \backslash\left[\lambda_{0}, \lambda_{\infty}\right]$. Consequently, $\theta_{A}(\lambda)$ is continuous on $\mathbf{R} \backslash\left[\lambda_{0}, \lambda_{\infty}\right]$ and in particular, taking account of (2.11), constant on each open intervals $\left(-\infty, \lambda_{0}\right)$ and $\left(\lambda_{\infty}, \infty\right)$. Furthermore, by the way of taking the branch of the logarithm and (2.9), we conclude the lemma.

Theorem 2.8. Let $V$ be a real-valued bounded function. Then,

$$
\frac{1}{|A|} \sum_{x \in G}\left(p^{V}(t, x, x)-p_{A}^{V}(t, x, x)\right)=e^{-\lambda_{\infty} t}+t \int_{\lambda_{0}}^{\lambda_{\infty}} e^{-\lambda t} \theta_{A}(\lambda) d \lambda
$$

where $\lambda_{0}\left(\right.$ resp. $\left.\lambda_{\infty}\right)$ is the minimum (resp. maximum) of the spectrum of $L$.

Proof. Since $p^{V}(t, x, x)$ is the kernel of the operator $e^{-t L}$, using the Dunford integral, we obtain the following expression:

$$
\begin{aligned}
& \sum_{x \in G}\left(p^{V}(t, x, x)-p_{A}^{V}(t, x, x)\right) \\
& =-\sum_{x \in G} \frac{1}{2 \pi i} \int_{C} e^{-\lambda t}\left(g_{\lambda}(x, x)-g_{\lambda}^{A}(x, x)\right) d \lambda
\end{aligned}
$$

where the contour $C$ is

$$
\begin{gathered}
\left\{\lambda_{0}-\delta+i \xi ;-\epsilon \leq \xi \leq \epsilon\right\} \cup\left\{\lambda_{\infty}+\delta+i \xi ;-\epsilon \leq \xi \leq \epsilon\right\} \\
\cup\left\{\xi \pm i \epsilon ; \lambda_{0}-\delta \leq \xi \leq \lambda_{\infty}+\delta\right\}
\end{gathered}
$$

for $\epsilon>0$ and $\delta>0$. The interchange of the summation and the integral over $C$ can be easily justified.

By Proposition 2. 6, we have

$$
\sum_{x \in G}\left(p^{V}(t, x, x)-p_{A}^{V}(t, x, x)\right)=\frac{-1}{2 \pi i} \int_{C} e^{-\lambda t} \frac{d}{d \lambda} \log \operatorname{det} G_{\lambda}^{A} d \lambda .
$$

Now we calculate the right-hand side.

$$
\begin{aligned}
& \frac{-1}{2 \pi i} \int_{C} e^{-\lambda t \frac{d}{d \lambda}} \log \operatorname{det} G_{\lambda}^{A} d \lambda \\
& =\frac{1}{\pi} \int_{\lambda_{0}-\delta}^{\lambda_{\infty}+\delta} \operatorname{Im}\left(e^{-(\lambda+\imath \epsilon) t} \frac{d}{d \lambda} \log \operatorname{det} G_{\lambda+i \epsilon}^{A}\right) d \lambda \\
& \quad+\frac{1}{2 \pi i} \int_{\lambda_{0}-\delta-\imath \epsilon}^{\lambda_{0}-\delta+\imath} e^{-\lambda t} \frac{d}{d \lambda} \log \operatorname{det} G_{\lambda}^{A} d \lambda \\
& +\frac{1}{2 \pi i} \int_{\lambda_{\infty}+\delta+\imath \epsilon}^{\lambda_{\infty}+\delta-\imath \epsilon} e^{-\lambda t \frac{d}{d \lambda} \log \operatorname{det} G_{\lambda}^{A} d \lambda}
\end{aligned}
$$


The second and third term of the right-hand side will vanish as $\epsilon \rightarrow 0$ since the integrands are analytic in the resolvent set. Integrating the first term by parts, we obtain

$$
\begin{aligned}
& \frac{1}{\pi} \int_{\lambda_{0}-\delta}^{\lambda_{\infty}+\delta} \operatorname{Im}\left(e^{-(\lambda+\imath \epsilon) t} \frac{d}{d \lambda} \log \operatorname{det} G_{\lambda+i c}^{A}\right) d \lambda \\
& =\frac{1}{\pi}\left[\operatorname{Im}\left(e^{-(\lambda+\imath \epsilon) t} \log \operatorname{det} G_{\lambda+\imath \epsilon}^{A}\right)\right]_{\lambda_{0}-\delta}^{\lambda_{\infty}+\delta} \\
& \quad+\frac{t}{\pi} \int_{\lambda_{0}-\delta}^{\lambda_{\infty}+\delta} \operatorname{Im}\left(e^{-(\lambda+\imath \epsilon) t} \log \operatorname{det} G_{\lambda+1 \epsilon}^{A}\right) d \lambda .
\end{aligned}
$$

Note that $\operatorname{Im} \log \operatorname{det} G_{\lambda}^{A}$ is bounded by (2.9). Using the dominated convergence theorem, as $\epsilon \longrightarrow 0$, we obtain

$$
\begin{aligned}
& \frac{1}{|A|} \sum_{x \in G}\left(p^{V}(t, x, x)-p_{A}^{V}(t, x, x)\right) \\
& =-e^{-\left(\lambda_{0}-\delta\right) t} \theta_{A}\left(\lambda_{0}-\delta\right)+e^{-\left(\lambda_{\infty}+\delta ! t\right.} \theta_{A}\left(\lambda_{\infty}+\delta\right) \\
& +t \int_{\lambda_{0}-\delta}^{\lambda_{\infty}+\delta} e^{-\lambda t} \theta_{A}(\lambda) d \lambda
\end{aligned}
$$

Hence, from Lemma 2. 7, as $\delta \rightarrow 0$, the proof is completed.

Theorem 2.9. Let $V$ be a real-valued bounded function. Then

$$
\frac{1}{|A|} \sum_{a \in A} V(a)=\lambda_{\infty}-1-\int_{\lambda_{0}}^{\lambda_{\infty}} \theta_{A}(\lambda) d \lambda
$$

Proof. Differentiating both sides of (2.12) and taking the limit $t \rightarrow 0$, we have the result because of Proposition 2.2.

\section{§3. An Example}

We will give an example which can be caiculated $\theta_{A}(\lambda)$ explicitly. This example is essentially due to Craig [2]. Let $G$ be a one-dimensional lattice $\mathbb{Z}^{1}$ and $A$ be a singleton $\{a\} . V$ is a $n$ periodic potential, that is, for fixed $n \geq 1$, $V(x)=V(y)$ if $d(x, y)=n$. In this case it is known that the spectrum of $L$ has a finite band structure. Precisely, the spectrum set is a union of finite closed intervals, for some $N$,

$$
\sigma(L)=\bigcup_{0 \leq k \leq N}\left[\lambda_{2 k}, \lambda_{2 k+1}\right]
$$


Also, the essential spectrum of $L_{a}$ is same as that of $L$ and the spectrum of $L_{a}$ may has eigenvalues. Since the green function $g_{\lambda}(a, a)$ is taking real value and monotone increasing on each resolvent set $I_{k}=\left(\lambda_{2 k-1}, \lambda_{2 k}\right)$, it has at most one zero on each $I_{k}$. If there exists a zero on $I_{k}$, we put it as $\mu_{k}(a)$ which is an eigenvalue of $L_{a}$. If $g_{\lambda}(a, a)>0 \quad($ resp. $<0)$ on $I_{k}$, we put $\mu_{k}(a)=\lambda_{2 k}$ (resp. $\left.\lambda_{2 k-1}\right)$.

Now we use much weaker version of the remarkable result in [6].

Theorem 3.1. Let $V$ be a periodic potential. Then, for a. e. $\lambda \in \sigma(L)$.

$$
\lim _{\epsilon \rightarrow 0} \operatorname{Re} g_{\lambda+\imath \epsilon}(a, a)=0 \text {. }
$$

For details, one may refer to $[6]$.

Now we can calculate $\theta_{A}(\lambda)$ as follows:

$$
\theta_{A}(\lambda)=\left\{\begin{array}{cl}
1, & \lambda_{2 k-1}<\lambda<\mu_{k}(a) . \\
0, & \mu_{k}(a)<\lambda<\lambda_{2 k} . \\
\frac{1}{2}, & \lambda_{2 k}<\lambda<\lambda_{2 k+1} .
\end{array}\right.
$$

It follows from Theorem 3. 1 and the fact $g_{\lambda}(a, a)$ is real and monotone increasing on the resolvent set. Then we have the following theorem:

Corollary 3.2. Let $G$ be $\mathbb{Z}^{1}$ and $V$ a periodic potential. Then

$$
V(a)=\frac{\lambda_{0}+\lambda_{\infty}}{2}-1+\frac{1}{2} \sum_{1 \leq k \leq N}\left(\lambda_{2 k-1}+\lambda_{2 k}-2 \mu_{k}(a)\right)
$$

Proof. By Theorem 2. 9 we have

$$
V^{\prime}(a)=\frac{\lambda_{0}+\lambda_{\infty}}{2}-1+\int_{\lambda_{0}}^{\lambda_{\infty}}\left(\frac{1}{2}-\theta_{A}(\lambda)\right) d \lambda
$$

Noting that $\frac{1}{2}-\theta_{A}(\lambda)$ vanishes on $\sigma(L)$, we have

$$
\begin{aligned}
V(a) & =\frac{\lambda_{0}}{2} \frac{+\lambda_{\infty}}{2}-1+\sum_{k=1}^{N} \int_{\lambda_{2 k-1}}^{\lambda_{2 k}}\left(\frac{1}{2}-\theta_{A}(\lambda)\right) d \lambda \\
& =\frac{\lambda_{0}+\lambda_{\infty}}{2}-1+\frac{1}{2} \sum_{k=1}^{N}\left(\lambda_{2 k-1}+\lambda_{2 k}-2 \mu_{k}(a)\right) .
\end{aligned}
$$

Remark 3.3. Corollary 3.2 also holds for so-called reflectionless potentials [2]. 


\section{§ 4. A Discrete Analogue of the Gutzwiller's Trace Formula}

Now in order to state a discrete analogue of the Gutzwiller's trace formula for open system, we define a function $d_{\lambda}$ on $V(G) \times V(G)$ as follows: for each $\lambda$ $\operatorname{Linf}_{x \in G} V(x)$

$$
d_{\lambda}(x, y)=-\frac{1}{2}\left(\log E_{x}\left[e^{-F_{\lambda}\left(T_{y}\right)}\right]+\log E_{y}\left[e^{-F_{2}\left(T_{x}\right)}\right]\right)
$$

where $F_{\lambda}(t)=F_{\lambda}(t, w)=\int_{0}^{t}\left(-\lambda+V\left(w_{t}\right)\right) d t$. Remark that since $g_{\lambda}(x, y)=$ $E_{x}\left[e^{-F_{\lambda}\left(T_{u}\right)} ; T_{y}<\infty\right] g_{\lambda}(y, y)$ and $E_{x}\left[e^{-F_{\lambda}\left(T_{y}\right)}\right]=E_{x}\left[e^{-F_{\lambda}\left(T_{y}\right)}: T_{y}<\infty\right]$ for $\lambda<\inf _{x \in G}$ $V(x)$,

$$
d_{\lambda}(x, y)=-\frac{1}{2} \log \frac{g_{\lambda}(x, y) g_{\lambda}(y, x)}{g_{\lambda}(x, x) g_{\lambda}(y, y)}
$$

Lemma 4.1. Let $\lambda<\inf _{x \in G} V(x)$. Then. $d_{\lambda}\left({ }^{\circ},{ }^{\circ}\right)$ is a distance, that is, $d_{\lambda}$ $(\circ, \circ): V(G) \times V(G) \rightarrow \mathbb{R}^{+}$satisfies the following:

(1) $d_{\lambda}(x, y) \geq 0$ and if $d_{\lambda}(x, y)=0$ then $x=y$,

(2) $d_{\lambda}(x, y)=d_{\lambda}(y, x)$,

(3) $d_{\lambda}(x, y) \leq d_{\lambda}(x, z)+d_{\lambda}(z, y)$.

Proof. (1) and (2) are trivial. So we will show the triangle inequality (3).

$$
\begin{aligned}
E_{x}\left[e^{\left.-F_{\lambda}, T_{2}\right)}\right] & =E_{x}\left[e^{-F_{\lambda}\left(T_{z}\right)} ; T_{y}<T_{z}\right]+E_{x}\left[e^{-F_{\lambda}\left(T_{z}\right)} ; T_{y}>T_{z}\right] \\
& =E_{x}\left[e^{-F_{\lambda}\left(T_{y}\right)} ; T_{y}<T_{z}, T_{y}<\infty\right] \cdot E_{y}\left[e^{-F_{\lambda}\left(T_{z}\right)}\right]+E_{x}\left[e^{-F_{\lambda}\left(T_{z}\right)} ; T_{y}>T_{z}\right] .
\end{aligned}
$$

Here we used the strong Markov property.

$$
\begin{aligned}
& -\log E_{x}\left[e^{-F_{\lambda}\left(T_{z}\right)}\right] \\
& \quad=-\log \left(E_{x}\left[e^{-F_{\lambda}\left(T_{y}\right)} ; T_{y}<T_{z}, T_{y}<\infty\right] \cdot E_{y}\left[e^{-F_{\lambda}\left(T_{z}\right)}\right]+E_{x}\left[e^{-F_{\lambda}\left(T_{z}\right)} ; T_{y}>T_{z}\right]\right) \\
& \leq-\log \left(E_{x}\left[e^{-F_{\lambda}\left(T_{y}\right)} ; T_{y}<T_{z}, T_{y}<\infty\right] \cdot E_{y}\left[e^{-F_{\lambda}\left(T_{x}\right)}\right]+E_{x}\left[e^{-F_{\lambda}\left(T_{y}\right)} ; T_{y}>T_{z}\right]\right) .
\end{aligned}
$$

Note that if $0<x, a, b \leq 1$ then $-\log (a x+b) \leq-\log (a+b)-\log x$. Then we have

$$
-\log E_{x}\left[e^{-F_{\lambda}\left(T_{z}\right)}\right] \leq-\log E_{x}\left[e^{-F_{\lambda}\left(T_{y}\right)}\right]-\log E_{y}\left[e^{-F_{\lambda}\left(T_{z}\right)}\right]
$$

Similarly, we have

$$
-\log E_{z}\left[e^{-F_{\lambda}\left(T_{x}\right)}\right] \leq-\log E_{z}\left[e^{-F_{\lambda}\left(T_{y}\right)}\right]-\log E_{y}\left[e^{-F_{\lambda}\left(T_{x}\right)}\right] .
$$

Then, we obtain the lemma.

It is easy to see that 


$$
d_{\lambda_{1}}>d_{\lambda_{2}} \text { if } \lambda_{1}<\lambda_{2}<\inf _{x \in G} V(x) .
$$

We are interested in the detailed asymptotic properties of the family of distances $\left\{d_{\lambda}\right\}$. However, we just give an easy example of $\left\{d_{\lambda}\right\}$ which can be explicitly calculated.

Example 4.2. Let $G$ be a $d$-regular tree and $V$ is identically zero. Let $\alpha_{d}=\frac{2 \sqrt{d-1}}{d}$. Then as is well known, the spectrum of $-\Delta_{G}$ is $\left[1-\alpha_{d}, 1+\alpha_{d}\right]$. By an easy calculation we obtain

$$
d_{\lambda}(x, y)=d(x, y) \cdot\left(-\log m_{d}(\lambda)\right)
$$

for $\lambda<0$. Here $d(x, y)$ is the same one defined by (2.2) and

$$
m_{d}(\lambda)=\frac{d}{2 d-2}\left(1-\lambda-\sqrt{(1-\lambda)^{2}-\alpha_{d}^{2}}\right) .
$$

Especially, as $\lambda \rightarrow 0$

$$
\begin{aligned}
& \text { (1) } \lim _{\lambda \rightarrow 0} d_{\lambda}(x, y)=d(x, y) \cdot \log (d-1) \quad \text { if } d \geq 3, \\
& \text { (2) } \lim _{\lambda \rightarrow 0-} \frac{d_{\lambda}(x, y)}{\sqrt{-2 \lambda}}=d(x, y) \quad \text { if } d=2
\end{aligned}
$$

and as $\lambda \rightarrow-\infty$

$$
d_{\lambda}(x, y) \sim d(x, y)\left\{\log (1-\lambda)+\log d-\frac{1}{4}\left(\frac{\alpha_{d}}{1-\lambda}\right)^{2}-\cdots\right\} .
$$

Now let us show a discrete version of the Gutzwiller's trace formula for our setting. Let $G_{\lambda}^{A}$ be the matrix that was defined in Lemma 2.3. We decompose $G_{\lambda}^{A}$ into two matrices $D_{\lambda}^{A}$ and $K_{\lambda}^{A}$ as follows:

$$
G_{\lambda}^{A}=D_{\lambda}^{A}\left(I+K_{\lambda}^{A}\right)
$$

where $D_{\lambda}^{A}$ is the diagonal matrix such that $\left(D_{\lambda}^{A}\right)_{a, a}=g_{\lambda}(a, a)$ for $a \in A$ and

$$
\left(K_{\lambda}^{A}\right)_{a, b}=\left\{\begin{array}{cc}
\frac{g_{\lambda}(a, b)}{g_{\lambda}(b, b)} & \text { if } a \neq b, \\
0 & \text { if } a=b .
\end{array}\right.
$$

Then,

Lemma 4.3. There exists $\tilde{\lambda} \in \mathbf{R}$ such that for any $\lambda<\tilde{\lambda}$

$$
\left\|K_{\lambda}^{\mathrm{A}}\right\|<1 \text {. }
$$


Proof. It is obvious by (2. 9).

Before we state our theorem, we prepare some notations. Let $\sigma$ be the shift transformation on $A^{\mathrm{N}}=\left\{\underline{a}=\left(a_{n}\right)_{n \in \mathrm{N}} ; a_{n} \in A\right\}$, i.e.,

$$
(\sigma \underline{a})_{n}=a_{n+1} \quad(n \in \mathbb{N}) \text {. }
$$

Let $\Sigma$ be the $\sigma$-invariant closed subset of $A^{\mathrm{N}}$ such that

$$
\Sigma=\left\{\underline{a} \in A^{\mathrm{N}} ; a_{n} \neq a_{n+1} \text { for any } n \in \mathbb{N}\right\} .
$$

The restriction of $\sigma$ on $\Sigma$ will be denoted again by $\sigma$. For a pair $(\Sigma, \sigma)$ we define

$$
\begin{aligned}
& F(n)=\left\{\underline{a} \in \sum ; \sigma^{n} \underline{a}=\underline{a}\right\} \\
& P(n)=F(n) \backslash \bigcup_{k \mid n} F(k)
\end{aligned}
$$

where $k \mid n$ means that $k$ is a divisor of $n$. For $\underline{a}, \underline{b} \in P(n)$ we define the equivalence relation by

$$
\underline{a} \sim \underline{b} \Leftrightarrow 0 \leq \exists k \leq n-1 \text { such that } \sigma^{k} \underline{a}=\underline{b} .
$$

Let $\Gamma_{n}=P(n) / \sim$ be the equivalence class of $P(n)$ by $\sim$. We call an element $\gamma$ of $\Gamma_{n}$ a prime periodic orbits with period $n$ and denote the period of $\gamma$ by $L_{\gamma}$. The totality of prime periodic orbits is denoted by $\Gamma$. Then, our theorem is the following:

Theorem 4.4. There exists $\tilde{\lambda} \in \mathbb{R}$ such that for any $\lambda<\tilde{\lambda}$

$$
\begin{aligned}
\sum_{x \in G}\left(g_{\lambda}(x, x)-g_{\lambda}^{A}(x, x)\right)= & \sum_{a \in A} \frac{d}{d \lambda} \log g_{\lambda}(a, a) \\
& +\sum_{r \in \Gamma} \frac{d S_{r}(\lambda)}{d \lambda} \sum_{n \geq 1} \exp \left(-n S_{r}(\lambda)-n \pi i L_{r}\right)
\end{aligned}
$$

where $S_{\gamma}(\lambda)$ is the length of a periodic orbit $\gamma$ with respect to the distance $d_{\lambda}$.

Proof. Since $\left\|K_{\lambda}^{A}\right\|<1$ for $\lambda<\tilde{\lambda}$, we have

$$
\begin{aligned}
\operatorname{det}\left(I+K_{\lambda}^{A}\right)=\operatorname{det} \exp \log \left(I+K_{\lambda}^{A}\right)= & \exp \left(\operatorname{Tr} \log \left(I+K_{\lambda}^{A}\right)\right) . \\
= & \exp \left(-\sum_{n=1}^{\infty} \frac{(-1)^{n}}{n} \operatorname{Tr}\left(K_{\lambda}^{A^{n}}\right)\right) .
\end{aligned}
$$

By the definition of $K_{\lambda}^{A}$, we obtain

$$
\operatorname{Tr}\left(K_{\lambda}^{4^{n}}\right)=\sum_{a_{1} a_{2}} \prod_{a_{n} \in F^{\prime}(n)} \prod_{i=1}^{n} E_{a_{1}}\left[e^{-F_{\lambda}{ }^{\prime} T_{a_{+1}}}\right]
$$


where $\dot{a}_{1} a_{2} \ldots \dot{a}_{n}$ is a periodic point and $a_{n+1}=a_{1}$. Noting that $S_{\gamma_{1} \gamma_{s}}(\lambda)=S_{\gamma_{1}}(\lambda)+$ $S_{\gamma_{2}}(\lambda)$ we obtain

$$
\begin{aligned}
& \operatorname{det}\left(I+K_{\lambda}^{A}\right)=\exp \left(-\sum_{n=1}^{\infty} \frac{1}{n} \sum_{\gamma \in F(n)} e^{-\left(S_{\wedge}(\lambda)+\imath \pi L_{2}\right)}\right) \\
& =\exp \left(-\sum_{n=1}^{\infty} \frac{1}{n} \sum_{\substack{\gamma \in P(k) \\
k ! n}} e^{-\frac{n}{k}\left(S_{.}(\lambda)+\imath \pi L_{\nu}\right)}\right) \\
& =\exp \left(-\sum_{k=1}^{\infty} \sum_{\gamma \in \Gamma,} \sum_{m=1}^{\infty} \frac{1}{m} e^{\left.-m \mid S_{\gamma}(\hat{\lambda})+1 \pi L_{\gamma^{\prime}}\right)}\right. \\
& =\prod_{\gamma \in \Gamma}\left(1-e^{-\left(S_{r}(\lambda)+2 \pi L_{r}\right)}\right) .
\end{aligned}
$$

Hence taking the logarithm and differentiating both sides of the equation above. we complete our proof.

Remark 4.5. For fixed $\lambda<\tilde{\lambda}$ the Fredholm determinant $\operatorname{det}\left(I-z K_{\lambda}^{A}\right)$ is the reciprocal of the Ruelle zeta function for the potential $U(\underline{a})=d_{\lambda}\left(a_{1}, a_{2}\right)+i \pi$. Here the Ruelle zeta function $\zeta(z)$ is defined by

$$
\zeta(z)=\exp \left(-\sum_{n=1}^{\infty} \frac{z^{n}}{n} \sum_{\underline{a} \in F^{\prime}(n)} e^{-S_{n} U^{\prime}(\underline{a})}\right)
$$

where $S_{n} L(\underline{a})=\sum_{k=0}^{n-1} U\left(\sigma^{k} \underline{a}\right)[1]$.

\section{References}

[1] Bowen. R., Equilibrium states and the ergodic theory of Anosov diffeomorphisms, Lecture Note in Mathematics, 470 (1975). Springer Verlag, Berlın.

[2] Craig, W., The Trace Formula for Schrödinger Operators on the Line, Comm. Math. Phy's, 126 (1989), 379-407.

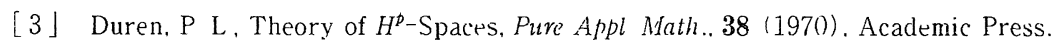

[4] Gesztesy, F., Holden, H. and Simon, B., Absolute Summability of the Trace Relation for Certain Schrödınger Operators. Comm. Math Phys, 168 (1995), 137-161.

[5] Gittwiller. M. C., Chans $m$ Clussical and Quantum Mcchanics, Springer Verlag. Berlın, 1990.

[6] Kotanı. S., One-Dimensıonal Random Schrödınger Operators and Herglotz Functions, Taniguch S.mp. PMMP Katata. (1985), 219-250

[7] Kotanı, S. and Simon, B.. Stochastic Schrödınger Operators and Jacobı Matrices on the Strıp. Comm. Math. Phys., 119 (1988), 403-429. 
